\title{
Improving SNR and reducing training time of classifiers in large datasets via kernel averaging
}

\author{
Matthias S. Treder ${ }^{1}$ \\ School of Computer Science \& Informatics, Cardiff University, Cardiff CF24 3AA, \\ United Kingdom \\ trederm@cardiff.ac.uk
}

\begin{abstract}
Kernel methods are of growing importance in neuroscience research. As an elegant extension of linear methods, they are able to model complex non-linear relationships. However, since the kernel matrix grows with data size, the training of classifiers is computationally demanding in large datasets. Here, a technique developed for linear classifiers is extended to kernel methods: In linearly separable data, replacing sets of instances by their averages improves signal-to-noise ratio (SNR) and reduces data size. In kernel methods, data is linearly non-separable in input space, but linearly separable in the high-dimensional feature space that kernel methods implicitly operate in. It is shown that a classifier can be efficiently trained on instances averaged in feature space by averaging entries in the kernel matrix. Using artificial and publicly available data, it is shown that kernel averaging improves classification performance substantially and reduces training time, even in non-linearly separable data.
\end{abstract}

Keywords: kernel $\cdot$ machine learning $\cdot$ big data $\cdot$ SVM $\cdot$ FDA

\section{Introduction}

In neuroscience, machine learning has been applied as a statistical tool for investigating brain activity or structure, and as a computational model emulating brain activity [10]. In the first capacity, it has mostly been employed in the form of classifiers for applications such as multivariate analysis [7], classification of clinical data [13], and brain-computer interfaces [11]. Multivariate analysis of neuroimaging data poses several challenges, two of which are addressed here. Firstly, signal-to-noise ratio (SNR) in neuroimaging data is typically low [8]. Secondly, the increasing use of large-scale datasets [3] leads to unprecedented statistical power but also high computational demands for training a classifier.

Cichy and Pantazis [4] presented a simple technique that addresses both challenges at once. They had participants view images repeated 30 times each while MEEG was recorded. Trials from the same class were randomly partitioned into groups of 5 , averaged to $l=30 / 5=6$ mean trials per class, and then used as input to a two-class linear Support Vector Machine (SVM). In [5] the authors averaged across groups of $l=40$ trials. Obviously, averaging reduces the size 
of the data by a factor of $l$, avoiding a bottleneck in classifier training time. In addition, averaging improves SNR substantially as evidenced by the high classification performance reported by the authors $[4,5]$.

As a linear operation, averaging only applies to linear classification tasks (see Fig. 1). Unfortunately, this precludes it from being used in kernel methods which can model more complex, non-linear relationships. Kernel methods are gaining popularity in social sciences [9] and in cognitive science [12], and they form the heart of the PRoNTo toolbox [15]. They are efficient when there is more variables than instances, such as in gene-expression data, since their complexity grows mainly with sample size $[18,14]$. More recently, multiple kernel learning has been explored for the classification of fMRI and EEG data $[20,16]$.

The purpose of this paper is to extend the instance averaging approach of $[4,5]$ to kernel methods. The basic idea is simple: kernel methods are non-linear in the original input space, but they act as linear models in a high-dimensional feature space. Therefore, instances can be averaged in feature space rather than in input space. It is shown that the necessary computations can be efficiently carried out in input space using the kernel trick. The benefits of kernel averaging are two-fold: a smaller kernel matrix allows for reduced classifier training times and less memory consumption. At the same time, the averaging increases the SNR of the data and hence classification performance.

The paper is structured as follows. At first, classification and averaging are formally introduced. The effect of data averaging is discussed for the input space and the feature space and an efficient computation using the kernel trick is presented. Subsequently, artificial data is used to illustrate that averaging instances in input space works for linearly separable data but not for linearly non-separable data, while kernel averaging works in both scenarios. Finally, four publicly available datasets are used to evaluate the effects of kernel averaging on classification performance and classifier training time.

\section{Method}

Let the $\mathrm{i}$-th instance in a dataset be denoted as $\mathbf{x}_{i} \in \mathcal{X}$, where $\mathcal{X}$ is the input space. Each instance has a corresponding class label $y_{i} \in \mathcal{Y}$, where $\mathcal{Y}$ is the set of class labels. A classifier is a function $f: \mathcal{X} \mapsto \mathcal{Y}$ that maps an instance onto a predicted class label. For a binary classification task with class labels +1 and -1 , any linear classifier can be written in the form

$$
f(\mathbf{x})=\operatorname{sgn}\left(\sum_{i} \alpha_{i} y_{i}\left\langle\mathbf{x}_{i}, \mathbf{x}\right\rangle\right)
$$

where the $\mathbf{x}_{i}$ 's are the training instances, $\mathbf{x}$ is a test instance, sgn is the signum function, and $\langle\cdot, \cdot\rangle$ is the standard dot product [14].

\subsection{The effect of averaging}

Let instances from a given class be drawn from a probability distribution $\mathcal{D}$ defined over $\mathcal{X}$, that is, $\forall i: \mathbf{x}_{i} \sim \mathcal{D}$. The average over $l$ instances converges 


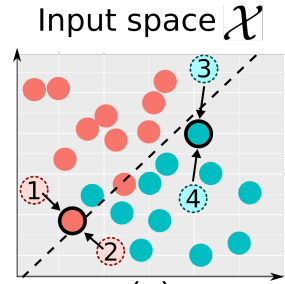

(a)

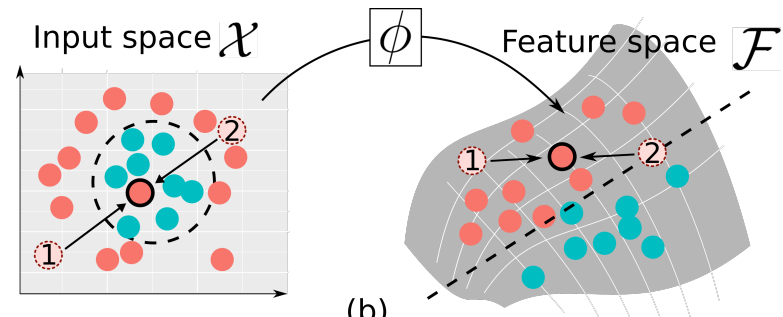

(b)

Fig. 1. Effect of averaging, illustrated for two classes (red and turquoise). (a) Linear classifiers use a linear decision boundary in input space (dashed line). When the red instances "1" and "2" are averaged (arrows), their average tends to be on the correct side of the hyperplane. The same applies to the turquoise instances "3" and "4". (b) Kernel classifiers use a non-linear decision boundary in input space (dashed circle). Averaging can pull the average onto the wrong side of the hyperplane even when the instances are on the correct side (see average of "1" and "2"). However, after projection into feature space using $\phi$, data are linearly separable.

towards the expectation $\mathbb{E}[\mathbf{x}]$ in probability, that is, $\frac{1}{l} \sum_{i=1}^{l} \mathbf{x}_{i} \stackrel{P}{\rightarrow} \mathbb{E}[\mathbf{x}]$ as $l \rightarrow \infty$. Does a classifier applied to averages perform better than a classifier applied to the original instances? If the support of $\mathcal{D}$ is a convex set, averaging can likely improve class separation (Fig. 1a). However, if it is non-convex, averaging can be detrimental. In extreme cases, the expected value occupies a part of input space wherein instances from this class are never observed, i.e. $P(\mathbf{x}=\mathbb{E}[\mathbf{x}])=0$. For instance, in Fig. 1b the turquoise class inhabits a circular region of input space. The region is convex so that averages remain within the circular region. However, the region inhabited by the red class is not convex and averages can end up within the circle although red instances are never observed there.

\subsection{Averaging instances in input space}

Cichy et al. $[4,5]$ averaged sets of instances prior to linear classification. Linear classifiers partition input space into convex regions so that averaging improves performance if the data is linearly separable. This can easily be demonstrated for the special case of a multivariate Gaussian distribution, i.e. $\mathbf{x}_{i} \sim \mathcal{N}(\mathbf{m}, \boldsymbol{\Sigma})$ with mean $\mathbf{m}$ and covariance matrix $\boldsymbol{\Sigma}$. The distribution of the average across $l$ instances is $\frac{1}{l} \sum_{i=1}^{l} \mathbf{x}_{i} \sim \mathcal{N}(\mathbf{m}, \mathbf{\Sigma} / l)$. In other words, the covariance matrix is scaled down by a factor of $l$. Since it is usually conceived of as the noise, averaged instances move closer to the class mean, increasing class separability. This approach is referred to as instance averaging in the rest of the paper.

\subsection{Averaging instances in feature space}

Kernel classifiers partition input space into potentially non-convex regions. The data is implicitly projected to a high-dimensional feature space $\mathcal{F}$ by applying a 
function $\phi: \mathcal{X} \mapsto \mathcal{F}$. A linear classifier is then applied in $\mathcal{F}$. The formula in Eq. (1) applies when replacing $\mathbf{x}$ by $\phi(\mathbf{x})$ :

$$
f(\mathbf{x})=\operatorname{sgn}\left(\sum_{i} \alpha_{i} y_{i}\left\langle\phi\left(\mathbf{x}_{i}\right), \phi(\mathbf{x})\right\rangle\right) .
$$

In other words, a kernel classifier acts as a linear classifier in feature space. Therefore, it is permissible to perform instance averaging in feature space. Next, an efficient way for averaging in feature space is developed.

If $\mathcal{F}$ is associated with a Reproducing Kernel Hilbert space, dot products in $\mathcal{F}$ can be represented by a kernel function $k: \mathcal{X} \times \mathcal{X} \mapsto \mathbb{R}, k\left(\mathbf{x}, \mathbf{x}^{\prime}\right)=\left\langle\phi(\mathbf{x}), \phi\left(\mathbf{x}^{\prime}\right)\right\rangle$. Crucially, this 'kernel trick' can also be used in order to train a classifier on averages of instances in feature space. To see this, let $\mathbf{z}=\left[\phi\left(\mathbf{x}_{1}\right)+\phi\left(\mathbf{x}_{2}\right)+\ldots+\right.$ $\left.\phi\left(\mathbf{x}_{l}\right)\right] / l$ and $\mathbf{z}^{\prime}=\left[\phi\left(\mathbf{x}_{1}^{\prime}\right)+\phi\left(\mathbf{x}_{2}^{\prime}\right)+\ldots+\phi\left(\mathbf{x}_{l}^{\prime}\right)\right] / l, l \in \mathbb{N}$ be two such averages. Then exploiting the bilinearity of the dot product one obtains

$$
\left\langle\mathbf{z}, \mathbf{z}^{\prime}\right\rangle=\frac{1}{l^{2}} \sum_{i, j \in\{1,2, . ., l\}}\left\langle\phi\left(\mathbf{x}_{i}\right), \phi\left(\mathbf{x}_{j}^{\prime}\right)\right\rangle=\frac{1}{l^{2}} \sum_{i, j \in\{1,2, . ., l\}} k\left(\mathbf{x}_{i}, \mathbf{x}_{j}^{\prime}\right) .
$$

Inserting this result into Eq. (2) yields a classifier trained on instances averaged in feature space. The quantity in Eq. (3) corresponds to a single entry in the averaged kernel matrix. It is obtained by averaging several entries in the full kernel matrix. Therefore, this approach is referred to as kernel averaging in the rest of the paper.

\subsection{Artificial data}
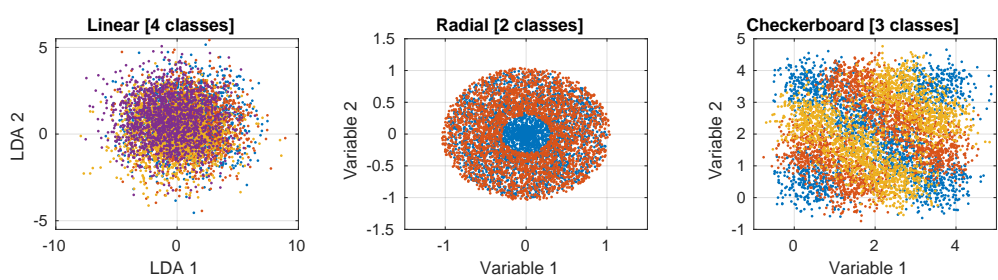

Fig. 2. Artificial datasets. The linear (Gaussian) dataset has been projected from 10 dimensions to the first 2 linear discriminant coordinates.

If the data is linearly separable, both instance averaging and kernel averaging should improve classification compared to no averaging. If the data is linearly non-separable, only kernel averaging should improve classification. To test this, three artificial datasets were generated and no averaging was compared to instance averaging and kernel averaging with groups of $l=50$ instances. 'Linear' data was sampled from a multivariate Gaussian distribution in 10 dimensions. 
Four classes were created with different means but an equal covariance matrix sampled from a Wishart distribution. Two non-linearly separable datasets were generated in two-dimensional input space, a 'radial' (2 classes) and a 'checkerboard' ( 3 classes) dataset. For each dataset, 6000 data points were created and Gaussian noise was added to every point. The datasets are depicted in Fig. 2.

\subsection{Real data}

To measure classification performance and computation time in real data, four publicly available datasets were analysed.

- EEG (http://bnci-horizon-2020.eu/database/data-sets). EEG from 11 participants performing an auditory oddball task [17]. Data were epoched in the interval $[-0.2,1] \mathrm{s}$ and baseline corrected. Non-EEG channels were removed. To create a large dataset, trials from all participants were pooled, yielding 25,247 trials (instances), 63 variables (EEG electrodes), 241 time points, and 2 classes. Data was averaged in the typical interval for the P300 component (300-500 ms).

- Gene expression (http://archive.ics.uci.edu/ml/datasets/gene+expression+cancer+RNA-

Seq). Part of the RNA-Seq (HiSeq) PANCAN dataset containing a random extraction of gene expressions of patients having different types of tumor [19]. It totals 801 instances, 20,531 variables, and 5 classes.

- p53 mutants (http://archive.ics.uci.edu/ml/datasets/p53+Mutants). The dataset models mutant p53 transcriptional activity (active vs inactive) based on data extracted from biophysical simulations [6]. It totals 16,772 instances, 5409 variables, and 2 classes.

- Cardiotocography (http://archive.ics.uci.edu/ml/datasets/Cardiotocography). Fetal cardiotocograms with classes corresponding to morphologic pattern [1]. It totals 2126 instances, 23 variables, and 10 classes.

\subsection{Analysis}

All analyses were conducted using MATLAB R2017a (Natick, USA) on an Intel Core i7-6700 CPU $3.40 \mathrm{GHz} \times 8$ computer with 64 GB RAM running on Ubuntu 18.04. Kernel Fisher Discriminant Analysis (FDA) and SVM were considered as classifiers. For kernel FDA, an open-source implementation (https://github.com/treder/MVPALight/tree/devel) was used. For SVM, LIBSVM [2] was used. LIBSVM does not implement kernel averaging, but it allows for pre-computed kernels to be provided. For this reason, the kernel matrix was pre-computed for all analyses and manually averaged. The same kernel with the same hyperparameters was used for kernel FDA and SVM. For the simulations and the EEG and gene expression datasets, an RBF kernel was used. For the p53 mutants and the Cardiotocography datasets, a polynomial kernel was used. Hyperparameters were fixed to gamma $=1 /$ \#variables, degree $=2$, and coef $0=0$. All variables were $\mathrm{z}$-scored.

Five-fold cross-validation was used to estimate classification accuracy. The procedure was repeated 10 times. Training time corresponds to the time it takes 
to train a single classifier in one fold. Classification accuracy and training time were averaged across folds and repetitions. Its standard deviation was also calculated across folds and repetitions.

\subsection{Complexity and memory}

Kernel averaging reduces the size of the kernel matrix by a factor of $l$. The complexity implications are discussed for kernel FDA and SVM. The most computationally demanding part of kernel FDA is an inversion and an eigenvalue decomposition of $n \times n$ matrices at a complexity of $\mathcal{O}\left(n^{3}\right)$. It is reduced to $\mathcal{O}\left(n^{3} / l^{3}\right)$ with kernel averaging. The complexity of the LIBSVM algorithm is reported as \#iterations $\times \mathcal{O}(n)$ if the $n \times n$ matrix can be cached [2]. Consequently, kernel FDA has a higher overall complexity than SVM and hence it should reap larger computational benefits from kernel averaging.

Memory consumption for storing the kernel matrix grows with $n^{2}$. For the largest dataset used in this paper (EEG data with 25,247 instances), the kernel matrix consumes about 2.3 GB RAM at single precision (4 bytes). The quadratic growth means that by just averaging 10 instances the memory required for the kernel matrix is reduced to about $23 \mathrm{MB}$, a factor of 100 smaller. The averaged kernel matrix can be built row-by-row or even entry-by-entry, so that the full kernel matrix is never actually required in memory.

\section{$3 \quad$ Results}
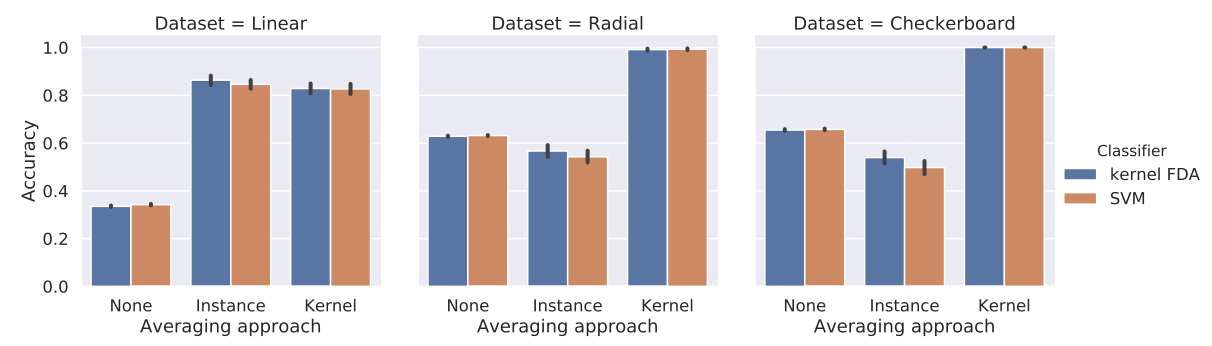

Fig. 3. Results on the artificial data. Similar results are obtained for kernel FDA (blue bars) and SVM (brown bars). When the data is linearly separable (Linear dataset), both instance averaging and kernel averaging improve classification performance. When the data is linearly non-separable (Radial and Checkerboard datasets), kernel averaging still improves classification performance but instance averaging does not.

Fig. 3 shows the results obtained on the artificial data. For linearly separable data, both instance averaging and kernel averaging improve classification performance from about $30 \%$ to about $80 \%$. However, for the Radial and Checkerboard 


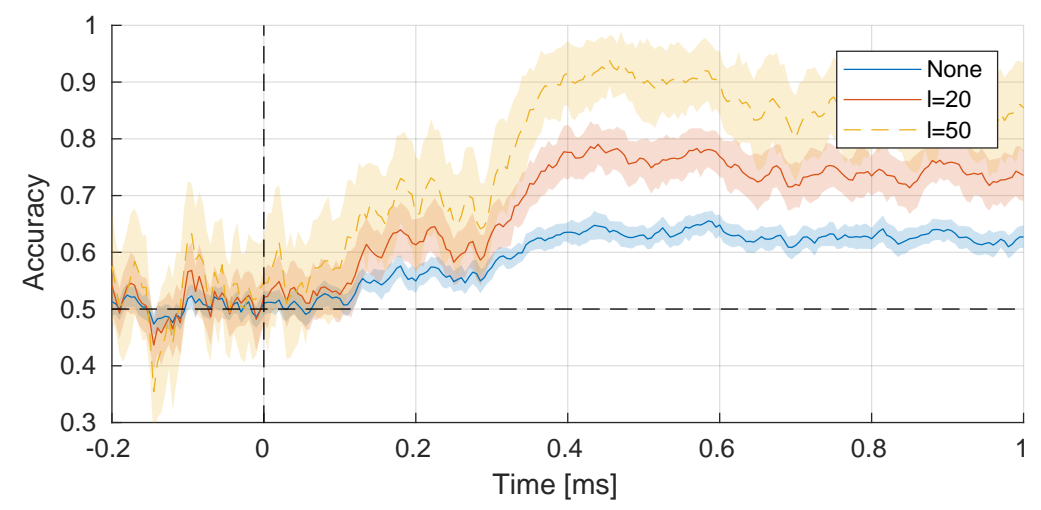

Fig. 4. Classification performance for participant \#1 of the EEG dataset. Classification accuracy is plotted for every time point, comparing no averaging and kernel averaging with $l=20$ and $l=50$.

data, which are linearly non-separable, only kernel averaging leads to an improvement from about $60 \%$ to almost $100 \%$. Instance averaging even has a slightly detrimental effect, leading to an accuracy $<60 \%$. Qualitatively, the same effects are observed for kernel FDA and SVM.

To illustrate the effect of kernel averaging on the EEG data, classification was performed for every time point for participant \#1 using kernel FDA, comparing no averaging to kernel averaging with $l=20$ and $l=50$. Results are depicted in Fig. 4. Clearly, kernel averaging improves classification performance. For the rest of the analyses, EEG data was averaged in the 300-500 ms interval and all participants were pooled. Table 3 depicts the results for the 4 datasets. In all cases, kernel averaging leads to a consistent improvement of classification performance. For the EEG data, accuracy increases from $62 \%$ to $99 \%$. For the Cardiotocography data, it increases from $88 \%$ to $98 \%$ (kernel FDA) and $91 \%$ to $99 \%$ (SVM). Even for the gene expression and p53 mutants datasets, kernel averaging improves performance although the classification is almost at ceiling to start with. The results are consistent across classifiers and kernels.

Kernel averaging also leads to a reduction of training time. This is especially evident for the two largest datasets, EEG and p53 mutants. In the EEG dataset, averaging $l=200$ instances reduces training time from $248.07 \mathrm{~s}$ to $5.78 \mathrm{~s}$ for kernel FDA $(40 \times$ faster), and from $15.67 \mathrm{~s}$ to 5.78 for SVM ( $3 \times$ faster). In the $\mathrm{p} 53$ mutants dataset, averaging $l=10$ instances reduces training time from $151.25 \mathrm{~s}$ to $3.42 \mathrm{~s}$ for kernel FDA ( $40 \times$ faster), and from $4.17 \mathrm{~s}$ to $3.1 \mathrm{~s}$ for SVM (1.3× faster). 


\begin{tabular}{|c|c|c|c|c|}
\hline Dataset & Classifier & $\begin{array}{c}\text { Kernel } \\
\text { averaging }\end{array}$ & $\begin{array}{c}\text { Classification } \\
\text { accuracy }\end{array}$ & $\begin{array}{l}\text { Training } \\
\text { time [sec] }\end{array}$ \\
\hline \multirow[t]{6}{*}{ EEG } & \multirow[t]{3}{*}{ Kernel FDA } & None & $.62 \pm .006$ & $248.07 \pm .49$ \\
\hline & & $l=50$ & $.96 \pm .02$ & $7.06 \pm .08$ \\
\hline & & $l=200$ & $.99 \pm .01$ & $5.78 \pm .12$ \\
\hline & \multirow[t]{3}{*}{$\overline{\mathrm{SVM}}$} & None & $.62 \pm .01$ & $15.67 \pm .26$ \\
\hline & & $l=50$ & $.96 \pm .02$ & $7.06 \pm .07$ \\
\hline & & $l=200$ & $.99 \pm .002$ & $5.78 \pm .16$ \\
\hline \multirow[t]{4}{*}{ Gene expression } & \multirow[t]{2}{*}{ Kernel FDA } & None & $.989 \pm .007$ & $.05 \pm .04$ \\
\hline & & $l=5$ & $1 \pm 0$ & $.01 \pm .01$ \\
\hline & \multirow[t]{2}{*}{ SVM } & None & $.988 \pm .007$ & $.008 \pm .003$ \\
\hline & & $l=5$ & $1 \pm 0$ & $.006 \pm .002$ \\
\hline \multirow[t]{6}{*}{ p53 mutant } & \multirow[t]{3}{*}{ Kernel FDA } & None & $.987 \pm .002$ & $151.25 \pm 25.52$ \\
\hline & & $l=5$ & $.993 \pm .003$ & $5.67 \pm 1.2$ \\
\hline & & $l=10$ & $.999 \pm .002$ & $3.42 \pm .7$ \\
\hline & \multirow[t]{3}{*}{ SVM } & None & $.9933 \pm .0013$ & $4.17 \pm .65$ \\
\hline & & $l=5$ & $.9989 \pm .0013$ & $4.03 \pm .67$ \\
\hline & & $l=10$ & $.9998 \pm .0004$ & $3.1 \pm .53$ \\
\hline \multirow[t]{4}{*}{ Cardiotocography } & \multirow[t]{2}{*}{ Kernel FDA } & None & $.88 \pm .02$ & $.635 \pm .141$ \\
\hline & & $l=5$ & $.98 \pm .01$ & $.075 \pm .024$ \\
\hline & \multirow[t]{2}{*}{$\overline{\mathrm{SVM}}$} & None & $.91 \pm .01$ & $.079 \pm .019$ \\
\hline & & $l=5$ & $.99 \pm .01$ & $.057 \pm .011$ \\
\hline
\end{tabular}

Table 1. Classification accuracy and training time for each of the 4 real datasets, classifier, and number of averages.

\section{Discussion}

An approach for averaging data in high-dimensional feature space has been developed. The analysis of the artificial data shows that instance averaging (in input space) fails to improve classification performance when the data is linearly non-separable. In contrast, kernel averaging (in feature space) improves classification performance for both linearly separable and non-separable data. This illustrates that kernel averaging is a genuine generalisation of averaging to nonlinear input spaces. In fact, instance averaging arises as a special case of kernel averaging when a linear kernel is used.

The analysis of 4 real datasets reveals a consistent improvement of classification performance by kernel averaging. Even classification on the gene expression and p53 mutants datasets improves significantly, despite the fact that performance is close to $100 \%$ to start with. Furthermore, there is a consistent reduction in training time. The benefit is most pronounced for kernel FDA with up to 40 times faster training on the EEG and p53 mutants datasets; smaller time benefits are obtained for SVM, in line with the complexity calculations.

Why not just subsample? For very large datasets, a simple alternative to kernel averaging is subsampling wherein just a subset of the data is selected. Because subsampling just discards large parts of the data, it does not offer the 
simultaneous improvement of SNR. However, if SNR is high to start with and performance is the only bottleneck, subsampling is a viable alternative.

How to interpret classification performance after averaging? If the classifier operates on averages rather than single instances, classification performance measures how well groups of instances can be classified. This nicely dovetails with many neuroscience applications. Often, a typical question is whether the classifier can differentiate between a patient group and a control group, or whether trials wherein participants viewed a face can be classified from trials wherein they viewed a scene. Whether or not a classifier trained using kernel averaging is also beneficial in the original, single-instance classification setting, is a separate question that will be addressed in future research.

How many instances should be averaged? The present results suggest that even for moderate values of $l$, such as 5 or 10, a significant improvement in class separability is achieved. For low SNR data such as EEG, larger values for $l$ such as 20 or 50 can yield improvement without reaching ceiling performance. Conversely, $l$ is bounded by data size. At least, $l$ should be small enough such that a handful of averaged samples of each class occur in every training set.

Concluding, a novel kernel averaging approach has been developed that generalises the notion of instance averaging to kernel classifiers. It allows for a significant improvement of SNR in conjunction with computational benefits, particularly for large datasets.

\section{References}

1. Ayres-de Campos, D., Bernardes, J., Garrido, A., Marques-de Sá, J., Pereira-Leite, L.: Sisporto 2.0: A program for automated analysis of cardiotocograms. The Journal of Maternal-Fetal Medicine 9(5), 311-318 (9 2000). https://doi.org/10.1002/1520-6661(200009/10)9:5i311::AID-MFM12_3.0.CO;2-9

2. Chang, C.c., Chang, C.c., Lin, C.j.: LIBSVM: A library for support vector machines. ACM Transactions on Intelligent Systems and Technology 2(3), 1-27 (2011)

3. Choudhury, S., Fishman, J.R., McGowan, M.L., Juengst, E.T.: Big data, open science and the brain: lessons learned from genomics. Frontiers in human neuroscience 8, 239 (2014). https://doi.org/10.3389/fnhum.2014.00239

4. Cichy, R.M., Pantazis, D.: Multivariate pattern analysis of MEG and EEG: A comparison of representational structure in time and space. NeuroImage 158, 441454 (9 2017). https://doi.org/10.1016/j.neuroimage.2017.07.023

5. Cichy, R.M., Ramirez, F.M., Pantazis, D.: Can visual information encoded in cortical columns be decoded from magnetoencephalography data in humans? NeuroImage 121, 193-204 (2015). https://doi.org/10.1016/j.neuroimage.2015.07.011

6. Danziger, S.A., Baronio, R., Ho, L., Hall, L., Salmon, K., Hatfield, G.W., Kaiser, P., Lathrop, R.H.: Predicting Positive p53 Cancer Rescue Regions Using Most Informative Positive (MIP) Active Learning. PLoS Computational Biology 5(9), e1000498 (9 2009). https://doi.org/10.1371/journal.pcbi.1000498

7. Dima, D.C., Perry, G., Singh, K.D.: Spatial frequency supports the emergence of categorical representations in visual cortex during natural scene perception. NeuroImage 179, $102-116 \quad\left(\begin{array}{ll}10 & 2018\end{array}\right)$. https://doi.org/10.1016/J.NEUROIMAGE.2018.06.033 
8. Gonzalez-Moreno, A., Aurtenetxe, S., Lopez-Garcia, M.E., del Pozo, F., Maestu, F., Nevado, A.: Signal-to-noise ratio of the MEG signal after preprocessing. Journal of Neuroscience Methods 222, 56-61 (1 2014). https://doi.org/10.1016/J.JNEUMETH.2013.10.019

9. Hainmueller, J., Hazlett, C., Alvarez, R.M.: Kernel Regularized Least Squares: Reducing Misspecification Bias with a Flexible and Interpretable Machine Learning Approach. Political Analysis 22(2), 143-168 (2014). https://doi.org/10.1093/pan/mpt019

10. Hinton, G.E.: Machine learning for neuroscience. Neural Systems \& Circuits 1(1), 12 (8 2011). https://doi.org/10.1186/2042-1001-1-12

11. Hwang, H.J., Ferreria, V.Y., Ulrich, D., Kilic, T., Chatziliadis, X., Blankertz, B., Treder, M.: A Gaze Independent Brain-Computer Interface Based on Visual Stimulation through Closed Eyelids. Scientific Reports 5, 15890 (10 2015). https://doi.org/10.1038/srep15890

12. Jäkel, F., Schölkopf, B., FA Wichmann: Does cognitive science need kernels? Trends in cognitive sciences (2009), https://www.sciencedirect.com/science/article/pii/S1364661309001430

13. Orrù, G., Pettersson-Yeo, W., Marquand, A.F., Sartori, G., Mechelli, A.: Using Support Vector Machine to identify imaging biomarkers of neurological and psychiatric disease: A critical review. Neuroscience \& Biobehavioral Reviews 36(4), 1140-1152 (4 2012). https://doi.org/10.1016/j.neubiorev.2012.01.004

14. Schölkopf, B., Smola, A.J.: A Short Introduction to Learning with Kernels. In: Advanced Lectures on Machine Learning, pp. 41-64. Springer, Berlin, Heidelberg (2003). https://doi.org/10.1007/3-540-36434-X_2

15. Schrouff, J., Rosa, M.J., Rondina, J.M., Marquand, A.F., Chu, C., Ashburner, J., Phillips, C., Richiardi, J., Mourão-Miranda, J.: PRoNTo: Pattern Recognition for Neuroimaging Toolbox. Neuroinformatics 11(3), 319-337 (7 2013). https://doi.org/10.1007/s12021-013-9178-1

16. Schrouff, J., Mourão-Miranda, J., Phillips, C., Parvizi, J.: Decoding intracranial EEG data with multiple kernel learning method. Journal of Neuroscience Methods 261, 19-28 (3 2016). https://doi.org/10.1016/J.JNEUMETH.2015.11.028

17. Treder, M.S., Purwins, H., Miklody, D., Sturm, I., Blankertz, B.: Decoding auditory attention to instruments in polyphonic music using single-trial EEG classification. Journal of neural engineering 11(2), 026009 (2014). https://doi.org/10.1088/1741$2560 / 11 / 2 / 026009$

18. Wang, X., Xing, E.P., Schaid, D.J.: Kernel methods for large-scale genomic data analysis. Briefings in bioinformatics 16(2), 183-92 (3 2015). https://doi.org/10.1093/bib/bbu024

19. Weinstein, J.N., Collisson, E.A., Mills, G.B., Shaw, K.R.M., Ozenberger, B.A., Ellrott, K., Shmulevich, I., Sander, C., Stuart, J.M., Stuart, J.M.: The Cancer Genome Atlas Pan-Cancer analysis project. Nature Genetics 45(10), 1113-1120 (10 2013). https://doi.org/10.1038/ng.2764

20. Youssofzadeh, V., McGuinness, B., Maguire, L.P., Wong-Lin, K.: Multi-Kernel Learning with Dartel Improves Combined MRI-PET Classification of Alzheimer's Disease in AIBL Data: Group and Individual Analyses. Frontiers in human neuroscience 11, 380 (2017). https://doi.org/10.3389/fnhum.2017.00380 